\title{
A new therapeutic strategy for PAH
}

Selective targeting of BMPR-II signalling using BMP9 could be a promising strategy for the treatment of pulmonary arterial hypertension (PAH), according to a new study. "This potential therapy targets the most common genetic cause of the disease - a pathway that is known to also be dysfunctional in non-genetic forms," says researcher Nicholas Morrell.

He explains that two major discoveries led to this work: the identification of mutations in BMPR2 in patients with PAH, and the observation that some individuals with ALK1 mutations develop severe PAH. "BMP9 and BMP10 are specific ligands for the BMPR-II/ALK-1 complex, which is almost exclusively found on endothelial cells. This finding led us to suggest that BMP9 (or BMP10) could be used to target the reduced function of BMPR-II/ALK-1 in PAH," says Morrell.

The researchers showed that BMP9 is highly specific for activation of BMP signalling in human endothelial cells. Moreover, BMP9 treatment protected cultured endothelial cells obtained from healthy individuals and from patients with BMPR2 mutations, against apoptosis, increased permeability and angiogenesis - key features of PAH pathobiology. Consistent with these findings, BMP9 prevented and reversed $\mathrm{PAH}$ in various rodent models, including the Sugenhypoxia model of severe disease and a novel knock-in model that carries a human disease-causing mutation in $B M P R 2$ and spontaneously develops PAH.

"BMP9 specifically targets the endothelium and is sufficient to reverse all the pathological changes in the pulmonary artery wall," concludes Morrell. "We are now looking to commercialise this discovery by optimizing BMP9 and BMP10 for use in the clinic."

Ellen F. Carney

Original article Long, L. et al. Selective enhancement of endothelial BMPR-II with BMP9 reverses pulmonary arterial hypertension. Nat. Med. doi:10.1038/nm.3877 